\title{
Hot stretch forming of titanium sheet by resistance heating
}

\author{
Xiaoqiang $\mathrm{Li}^{1 *}$, Hongrui Dong ${ }^{1}$, Guiqiang $\mathrm{Guo}^{1}$, and Dongsheng $\mathrm{Li}^{1}$ \\ ${ }^{1}$ School of Mechanical Engineering \& Automation, Beihang University, 100191 Beijing, China
}

\begin{abstract}
Titanium alloys are important aerospace materials due to excellent comprehensive performance, but it is difficult to form at room temperature. Electrically assisted hot stretch forming is used to form the titanium panels of aircraft. This paper studies the effect of temperature, strain, stress relaxation time and cooling rate on forming accuracy by stress relaxation test, tensile test and forming experiment. It is shown that for the influence of temperature on forming accuracy, there is no large difference among $773 \mathrm{~K}, 873 \mathrm{~K}, 973 \mathrm{~K}$. And with increase of strain, the forming accuracy becomes lower. For the influence of stress relaxation time, there is no large difference among $0 \mathrm{~min}, 15 \mathrm{mins}, 30 \mathrm{mins}$. And with decrease of cooling rate, the forming accuracy is improved significantly. It's concluded that when the temperature reaches a high level, temperature and stress relaxation time have no great influence on forming accuracy. And the forming accuracy is mainly influenced by strain and cooling rate. Electrically assisted hot stretch forming is an effective method to form titanium alloy. And the part with high forming accuracy can be obtained by adopting high temperature, low strain, proper stress relaxation time and low cooling rate.
\end{abstract}

Keyword: Hot stretch forming, stress relaxation, cooling rate

\section{Introduction}

With the growing use of composites in aircraft transport, titanium alloys are widely used in aerospace due to their high strength to weight ratio, perfect compatibility with composite materials and excellent corrosion resistance.[1] These components are typical extrude shape, which used to be made with aluminum and be formed by stretching at room temperature.[2] But titanium alloys are difficult to form due to their poor formability and large springback at room temperature. In order to improve the formability and decrease the springback, hot forming is used to form the titanium panels of aircraft. However, traditional heating method by furnace has the shortcoming of low efficiency and high consumption and requires complex equipment. So in order to improve heating efficiency and simplify experimental equipment, many other assisted heating methods are put forward, such as laser and electricity.

Among all titanium alloys, Ti-6.5Al-2Zr-1Mo-1V is a kind of near $\alpha$ titanium with high $\mathrm{Al}$ equivalent. It is used to manufacture bulkhead, panel and other important structural parts due to its attractive properties such as high specific strength, thermal stability and welding ability.[3][4] For the manufacturing of bulkhead, a new process of electrically assisted hot stretch forming is put forward, which combines traditional stretch forming with hot titanium forming technique and improves formability and forming accuracy by self-resistance heating. This technique saves material and machining time, which are two serious cost issues for today's aircraft manufacturers. And this process consists of four stages: heating sheet, stretching forming, stress relaxation and cooling, and the corresponding process parameters at each stage are temperature, strain, stress relaxation time and cooling rate respectively, as shown in Fig.1. Because bulkheads are usually slender members with curvature and forming quality of these components directly affects the assembly precision, the aerodynamic shape and the service life of aircraft, bulkheads with high quality need to be obtained.[5]

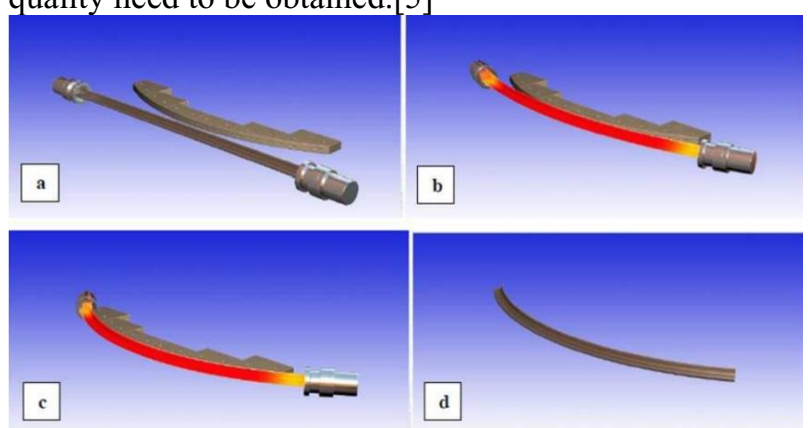

Fig.1 Process procedure of electrically assisted hot stretch forming [2]

A.Astarita et al. studied metallurgical aspect of hot stretch forming by microstructure experiment and the stress and strains by establishing a finite element model of Ti-6Al-4 extrusion in DEFORM.[2] The residual stresses after stretch bending and creep forming period were predicted. A lower stress distribution was in the middle of the extrusion while a contrary trend occurred

\footnotetext{
* Corresponding author: lixiaoqiang@buaa.edu.cn
} 
on the side because of the effect of the clamps. And the stress was reduced and became more homogeneous after the creep forming period. Deng et al. created a hot stretch forming model in ABAQUS to research the effect of dwell time on spring-back of Ti-6Al-4V and OT4 extrusion during creep forming period. The results indicated that the spring-back decreased greatly with the increase of dwell time.[6] Above previous researches didn't consider the effect of strain, which has great effect on residual stress and springback. And different stages interact. For example, stress relaxation exists in the process of stretching forming. So it's necessary to study the effects of these parameters on forming accuracy and determine which parameter is the main effect factor.

This paper studies the stress relaxation and hot stretch behaviour of $\mathrm{Ti}-6.5 \mathrm{Al}-2 \mathrm{Zr}-1 \mathrm{Mo}-1 \mathrm{~V}$ and the effects of temperature( $773 \mathrm{~K}, 873 \mathrm{~K}, 973 \mathrm{~K})$, strain $(0 \%$, $2 \%, 4 \%)$, stress relaxation time( $0 \mathrm{~min}, 15 \mathrm{mins}, 30 \mathrm{mins})$ and cooling $\mathrm{rate}(80 \mathrm{~K} / \mathrm{min}, 120 \mathrm{~K} / \mathrm{min}, 160 \mathrm{~K} / \mathrm{min})$ on forming accuracy.

\section{Experiment}

\subsection{Test material and designed parts}

The test material is Ti-6.5Al-2Zr-1Mo-1V and its chemical composition is listed in table 1 . The asreceived alloy was annealed after hot and cold rolling into $1 \mathrm{~mm}$-thick sheet. And the geometry of stress relaxation specimen is as shown in Fig.2

Table.1 The chemical composition of Ti-6.5Al-2Zr-1Mo-1V

\begin{tabular}{ccccccc|c}
\multicolumn{7}{c}{ alloy $(\%)$} \\
\hline $\mathrm{Al}$ & $\mathrm{Zr}$ & $\mathrm{Mo}$ & $\mathrm{V}$ & $\mathrm{Si}$ & $\mathrm{Fe}$ & $\mathrm{Ti}$ \\
\hline $5.5-7.0$ & $1.5-2.5$ & $0.5-2.0$ & $0.8-2.5$ & $\leq 0.15$ & $\leq 0.25$ & $\mathrm{Bal}$ \\
\hline & & & & & & & \\
\hline & & & & & & & \\
\hline
\end{tabular}

Fig.2 Geometry of stress relaxation specimen

Based on the existing die and equipment, the designed part and die are as shown Fig.3. The material of forming sheet is Ti-6.5Al-2Zr-1Mo- $1 \mathrm{~V}$, and the material of die is self-designed lime cement plate, which can withstand a very high temperature. The corresponding dimensions are as shown in Fig.3.(a). And the diagrams of geometrical position before forming and after forming are as shown in Fig.3.(b) and Fig.3.(c) respectively.

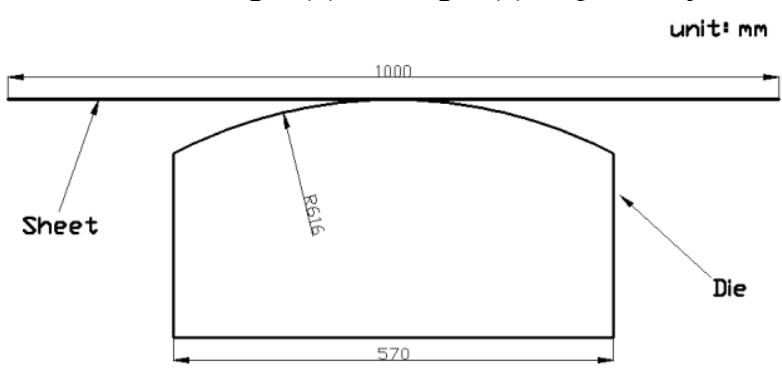

(a)

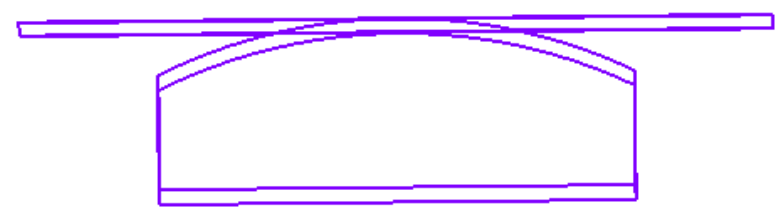

(b)

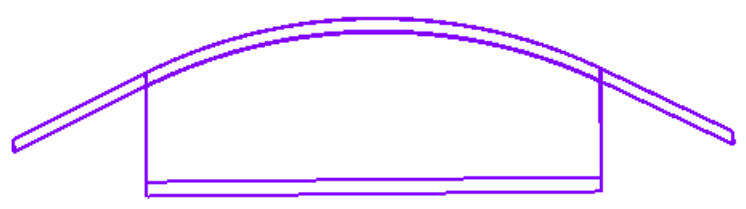

(c)

Fig.3. Schematic of designed part and die (a).The geometry of part and die (b).The diagram of geometrical position before forming (c). The diagram of geometrical position after forming

Because the effect of strain will be studied and the stress does not always have positive correlation with strain under high temperature, it is need to study tensile behaviour simply. The geometry of tensile specimen is as shown in Fig.4.

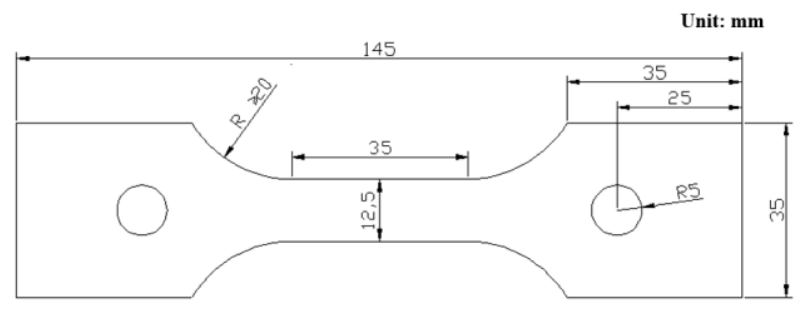

Fig.4 Geometry of tensile specimen

\subsection{Experiment equipment and procedure}

The stress relaxation experiments were conducted by using a Zwick/Roell Z100 electric universal test machine equipped with a heating furnace. The furnace offered a constant temperature zone with $200 \mathrm{~mm}$ length and the possibility to control the atmosphere temperature with \pm $3 \mathrm{~K}$ precision by three independent heating zones. The designed test matrix about stress relaxation is as shown in table.2. Firstly, the specimen was heated to the desired temperature and then kept warm to make temperature uniform. Secondly, stretched the specimen to the pre strain $10 \%$. Finally, fixed the clamp and recorded timestress curve for $60 \mathrm{mins}$.

Table.2 The designed test matrix about stress relaxation

\begin{tabular}{lll}
\hline Temperature & Pre-strain & Stress relaxation time \\
\hline $773 \mathrm{~K}$ & $10 \%$ & $60 \mathrm{mins}$ \\
$873 \mathrm{~K}$ & $10 \%$ & $60 \mathrm{mins}$ \\
$973 \mathrm{~K}$ & $10 \%$ & $60 \mathrm{mins}$ \\
\hline
\end{tabular}

In order to study the effects of temperature, strain, stress relaxation time and cooling rate on forming accuracy, the designed experiment matrix is as shown in table 3 .

Table.3 Experiment matrix about electrically assisted hot stretch forming

\begin{tabular}{ccccc}
\hline & $\begin{array}{c}\text { Temperature } \\
(\mathbf{K})\end{array}$ & Strain & $\begin{array}{c}\text { Stress } \\
\text { relaxation } \\
\text { time } \\
(\mathbf{m i n})\end{array}$ & $\begin{array}{c}\text { Cooling rate } \\
(\mathrm{K} / \mathbf{m i n})\end{array}$ \\
\hline Effect of & 773 & $2 \%$ & 15 & 120 \\
temperature & 873 & $2 \%$ & 15 & 120 \\
\hline Effect of strain & 973 & $2 \%$ & 15 & 120 \\
\hline
\end{tabular}




\begin{tabular}{ccccc}
\hline & 873 & $2 \%$ & 15 & 120 \\
& 873 & $4 \%$ & 15 & 120 \\
\hline Effect of stress & 873 & $2 \%$ & 0 & 120 \\
relaxation time & 873 & $2 \%$ & 15 & 120 \\
\hline \multirow{2}{*}{ Effect of } & 873 & $2 \%$ & 30 & 120 \\
cooling rate & 873 & $2 \%$ & 15 & 80 \\
\hline
\end{tabular}

The experiments were made with 50 tons stretching machine and an incubator, in which there is a die, as shown in Fig.5. Sheet was heated by self-resistance. The temperature was measured by thermocouple and controlled by closed loop control system.

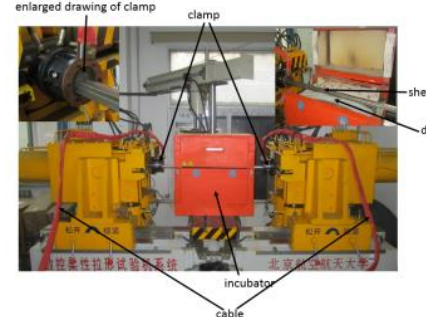

(a)

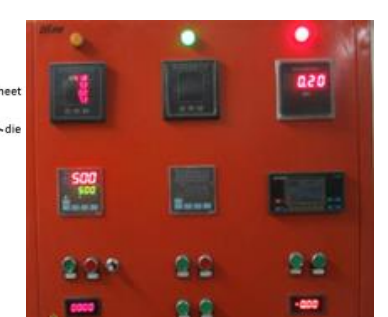

(b)

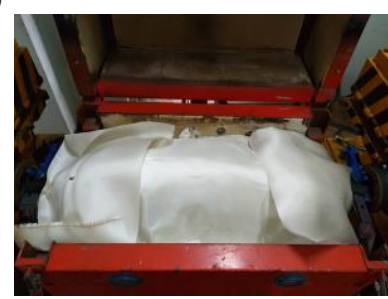

(c)

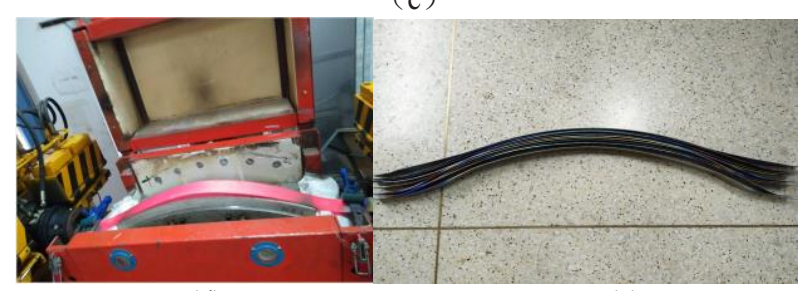

(d)

(e)
Fig.5. Diagram of stretching machine

(a). Stretching machine (b). Temperature control system (c).

Incubator with insulation cotton (d). Picture in forming (e). Formed part

Firstly, the specimen was put into incubator to control temperature by closed-loop control of current and the blank was heated by electricity current to temperature 773K, 873K, 973K. During forming process, the temperatures remains constant. Secondly, the blank was stretched by the lifting of die to strain $0 \%$, $2 \%, 4 \%$. Thirdly, the stress was relaxed by fixing incubator and clamp for $0 \mathrm{~min}, 15 \mathrm{mins}$, 30mins. Finally, the blank cooled at the cooling rate of $80 \mathrm{~K} / \mathrm{min}$, $120 \mathrm{~K} / \mathrm{min}, 160 \mathrm{~K} / \mathrm{min}$.

After the specimens were formed, the profiles of formed specimens were measured with three coordinate measuring machine, as shown in Fig.6.

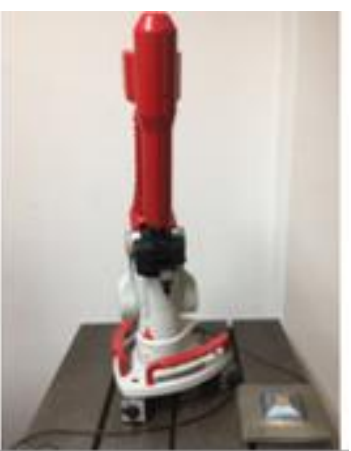

Fig.6 Diagram of three coordinate measuring machine

The tensile experiment was conducted with the same equipment as stress relaxation experiment. The designed test matrix is as shown in table.4. The specimen was heated to temperature $873 \mathrm{~K}$ and then stretched until it cracked.

Table.4 Experiment matrix about tensile experiment

\begin{tabular}{ll}
\hline Temperature & Strain rate \\
\hline $873 \mathrm{~K}$ & $0.001 / \mathrm{s}$ \\
\hline
\end{tabular}

\section{Result and discussion}

\subsection{Result and discussion of stress relaxation test}

Fig.7 is recorded time-stress curve of Ti-6.5Al-2Zr-1Mo$1 \mathrm{~V}$ at $773 \mathrm{~K}, 873 \mathrm{~K}, 973 \mathrm{~K}$ in stress relaxation tests. As shown in Fig.7, the stress relaxation curve can be divided into three parts. At first, stress declines rapidly. Subsequently, the stress relaxation goes into the transition stage. Finally, the stress relaxation goes into an apparent stable stage. It can be concluded that the increase of temperature is beneficial to accelerate the decrease of stress, which is because high temperature is beneficial to accelerate dynamic recovery and dynamic recrystallization. In order to quantify stress relaxation efficiency under different experimental condition, stress relaxation ratio (SRS) is introduced, which is expressed as Eq.1.[7]

$$
\Delta=\frac{\sigma}{\sigma_{0}}
$$

where $\sigma$ is instantaneous stress value and $\sigma_{0}$ is initial stress value.

Ultimate SRS values of $773 \mathrm{~K}, 873 \mathrm{~K}, 973 \mathrm{~K}$ are $50.8 \%, 13.4 \% .2 \%$ respectively. It indicates that with increase of temperature, the residual stress decreases and elastic strain can be transformed into plastic strain more thoroughly, which is helpful to reduce springback. 


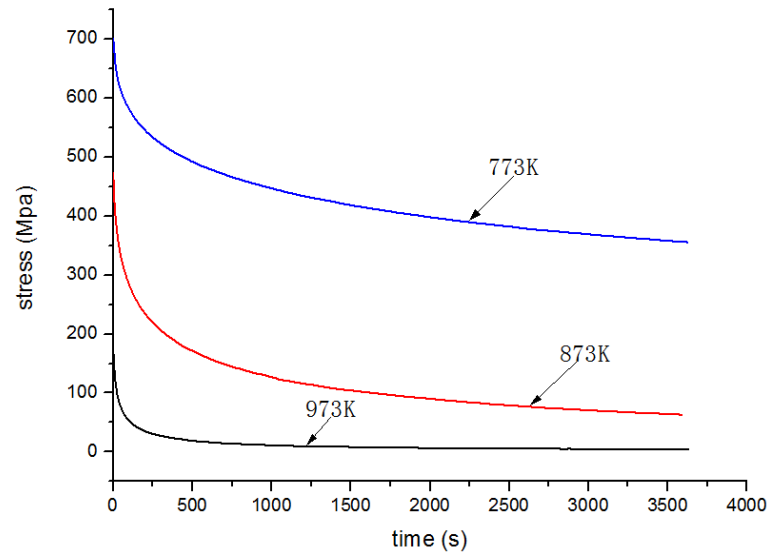

Fig.7 Stress relaxation curves of Ti-6.5Al-2Zr-1Mo-1V at $773 \mathrm{~K}, 873 \mathrm{~K}, 973 \mathrm{~K}$

\subsection{Result and discussion of tensile test}

Fig. 8 is recorded true stress-true strain curve of Ti$6.5 \mathrm{Al}-2 \mathrm{Zr}-1 \mathrm{Mo}-1 \mathrm{~V}$ at $873 \mathrm{~K}$ in the process of hot tensile test. As shown in Fig. 8 , when strain reaches $2 \%$ and 4\%, the material has got into plastic stage. And stress value of strain $4 \%$ is larger than stress value of strain $2 \%$, which indicates that hardening effect is still dominant when the forming temperature is $873 \mathrm{~K}$. So for the initial stress value in the process of stress relaxation, the value of $4 \%$ is larger than the value of $2 \%$.

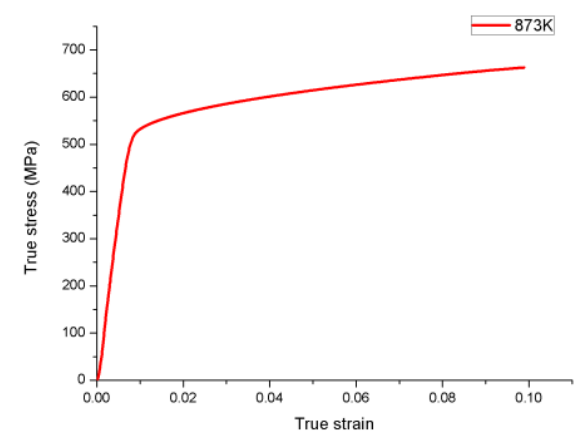

Fig.8. True stress-true strain curve of Ti-6.5Al-2Zr-1Mo-1V at $873 \mathrm{~K}$ in the process of hot tensile test

\subsection{Result and discussion of forming experiment}

The profiles of formed part under different conditions are shown in Fig.9. In Fig.9, the first parameter 873 indicates temperature $873 \mathrm{~K}$, the second parameter 2 indicates strain $2 \%$, the third parameter 15 indicates stress relaxation time $15 \mathrm{mins}$, the last one 120 indicates cooling rate $120 \mathrm{~K} / \mathrm{min}$, and they are expressed as $873-2$ 15-120.

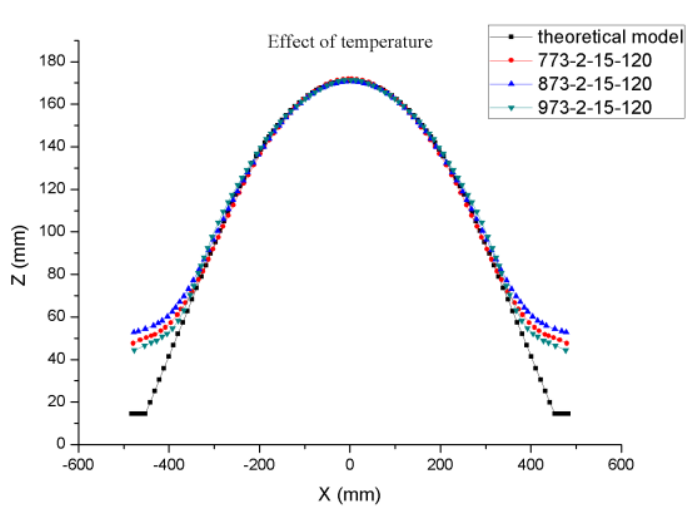

(a)

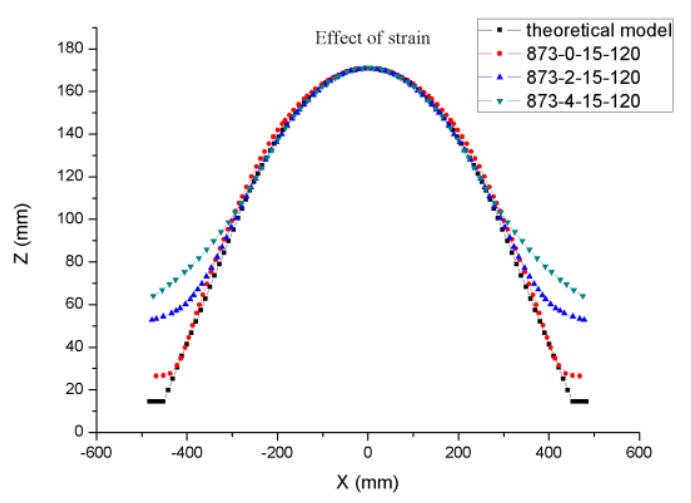

(b)

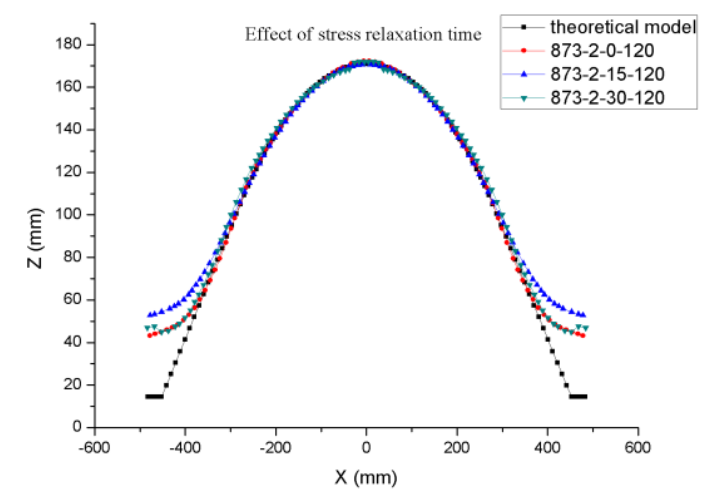

(c)

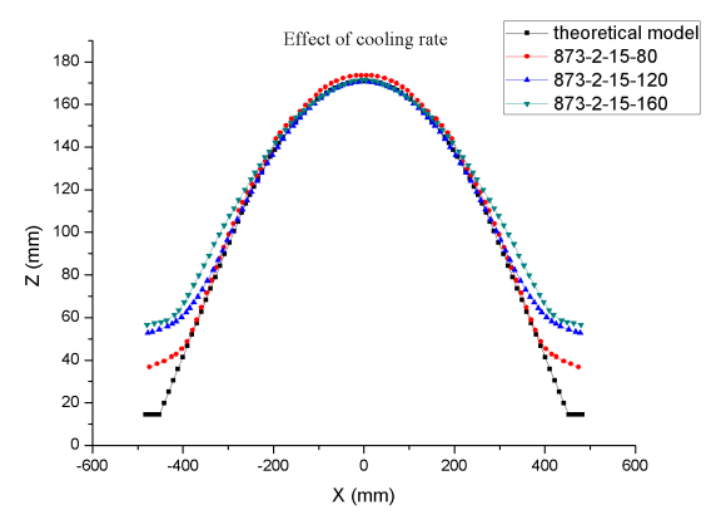

(d)

Fig.9. The profiles of formed part under different conditions (a) The profiles under different temperature 
(b) The profiles under different strain

(c) The profiles under different stress relaxation time

(d) The profiles under different cooling rate

For the effect of temperature, as shown in Fig.9(a), when stress relaxation time and cooling rate are proper, there is not large difference among $773 \mathrm{~K}, 873 \mathrm{~K}, 973 \mathrm{~K}$. It is concluded that when the temperature reaches a high level, it will have no great influence on forming accuracy of arc section. But for the whole section, forming accuracy of $973 \mathrm{~K}$ is the highest, which is because with increase of temperature, the residual stress decreases.

For the effect of strain, as shown in Fig.9(b), with increase of strain, both of the forming accuracies of arc section and the whole section decrease. This is because with increase of strain, the corresponding stress increases after stretching, and hardening effect is dominant, which results in higher residual stress after stress relaxation and larger springback after unloading.

For the effect of stress relaxation time, as shown in Fig.9(c), when temperature, strain and cooling rate are proper, there is not large difference among $0 \mathrm{~min}, 15 \mathrm{mins}$, 30 mins. It should have been that stress relaxation time had the largest effect on forming accuracy, which is because stress relaxation time effects the residual stress greatly, but that's not the case. This is because in the process of heating, stretching, relaxation stress and cooling, all of them effect the residual stress level. When the temperature reaches $873 \mathrm{~K}$ and the cooling rate is controlled properly, the residual stress is close for different stress relaxation time.

For the effect of cooling rate, as shown in Fig.9(d), with increase of cooling rate, the forming accuracy decreases, which is because residual stress increases with increase of cooling rate.

\section{Conclusion}

1. With increase of temperature, the residual stress decreases and elastic strain can be transformed into plastic strain more thoroughly, which is helpful to reduce springback.

2. When the sheet is formed under high temperature, besides in the process of stress relaxation, there is still stress relaxation behaviour in the process of forming and cooling rate, which has great influence on the forming accuracy.

3. When the temperature reaches a high level, temperature and stress relaxation time have no great influence on forming accuracy. And when the temperature reaches a high level, the forming accuracy increases significantly with decrease of strain and cooling rate.

4. Electrically assisted hot stretch forming is an effective method to form titanium alloy which is difficult to form under room temperature. And the part with high forming accuracy can be obtained by adopting high temperature $(>773 \mathrm{~K})$, low strain, proper stress relaxation time and low cooling rate.

\section{References}

1. M. Peters, C. Leyens, Titanium and Titanium Alloys - Fundamentals and Applications (2003)

2. A. Astarita, E. Armentani, E. Ceretti, L. Giorleo, P. Mastrilli, Key. Eng. Mater, 554-557,647-656 (2003)

3. P. Yao, P. Li, C. Li, K. Xue, G. Gan, Chin. J. Rare. Met, 39, 967-974 (2015)

4. W. Cheng, H. Yang, X. Fan, Z. Sun, Trans. Nonferrous Met. Soc. China, 21. 1963-1969 (2011)

5. G. Guo, D. Li, X. Li, et al, Int. J. Adv. Manuf. Technol, 1-2,1-13 (2017).

6. T. Deng, D. Li,, X. Li, P. Ding, K. Zhao, P. I. Mech. Eng. B-J. Eng, 230, 505-516 (2016)

7. Y. Liu, J. Zhu, Mech Mater, 40, 792-795 (2008) 